\title{
A high-accuracy indoor positioning system based on UWB
}

\author{
Hanyu Liu, Yanhan Zeng *, Ruguo Li and Huajie Huang \\ School of Physics \& Electronic Engineering, Guangzhou University Guangzhou, Guangdong, China
}

\begin{abstract}
In this paper, a high-accuracy indoor positioning system based on the ultra-wideband (UWB) technique is proposed. The proposed system uses a simple ranging process to obtain the distance between the mobile node and the fixed base stations. Besides, an improved time of arrival (ToA) algorithm with Kalman filtering is proposed to improve the positioning accuracy. Measurements have been performed in the real indoor $13 \mathrm{~m} * 7.6 \mathrm{~m}$ environment with many obstacles and the root-mean-square error (RMSE) is less than $0.3 \mathrm{~m}$. The pro- posed system offers a wide range of application in robotics, industrial automation, postsorting system and so on.
\end{abstract}

\section{Introduciton}

Localization systems with high accuracy is urgently needed for both commercial and governmental interests, with the popular of location-based services [2]. According to geolocation information, localization techniques can be classified into two categories: outdoor localization and indoor localization. Outdoor technologies like Global Positioning System (GPS), has been applied into many applications. But they cannot provide accurate indoor geolocation, which has its own independent market and unique technical challenges [12]

Many solutions have been proposed based on various kind- $\mathrm{s}$ of information, e.g. ToA $[1,6,11]$, time difference of arrival (TDoA) [5], phase of arrival (PoA) [3], phase difference of arrival (PDoA) [13], received signal strength indicator (RSSI) [7], etc. Localization algorithms can be simply classified in- to two categories: triangulation-based methods and pattern matching based methods.

The major challenge in the indoor positioning is that there are many obstacles. RSSI algorithm [10] is difficult to distinguish the small angle change of the mobile nodes because of the multipath disturbance. [9] uses a frequency modulated continuous wave (FMCW) based signal as the carrier but can only work well under the ideal fading model. [8] positioning is achieved by combining vector network analyser with the angle of analysis signal wave, which requires special signal angle antenna. [4] obtains a positioning accuracy less than $1.68 \mathrm{~m}$, but the bandwidth of $2 \mathrm{GHz}$ is required.

Due to the ability to penetrate obstacles and fine ranging resolution, UWB offers great advantage for achieving high positioning accuracy in indoor environments [2]. In this paper, an indoor positioning system with improved ToA based on UWB is proposed. The paper is organized as follows. To begin with, the system including the UWB signal and the ranging method is described in section 2 . Then we describe the improved positioning algorithm with Kalman filtering in section 3 . In section 4 , both the motion trajectory and the positioning accuracy are measured to verify the performance of proposed positioning system. Finally, the conclusion is drawn in section 5 .

\section{System Design}

\subsection{The UWB signal}

American Communications Commission officially decided in 2002 to open up the $3.1 \mathrm{GHz}-10.6 \mathrm{GHz}$ spaces for UWB to be used for the indoor communication. Federal Communication Commission (FCC) defines the UWB signal as:

$$
\frac{\left(f_{\mathrm{H}}-f_{\mathrm{L}}\right)}{f_{c}} \geq 20 \% \text { or } f_{\mathrm{H}}-f_{\mathrm{L}} \geq 500 \mathrm{MHz}
$$

Where $f_{H}$ and $f_{L}$ and are the upper frequency and lower frequency, when the peak power decreases to $10 \mathrm{~dB}$, respectively. And $f_{C}=\frac{f_{H}+f_{L}}{2}$ is the center frequency. According to Shannon's theorem, the large channel bandwidth can improve the capacity of the channel. The signal bandwidth of UWB system can be as high as $500 \mathrm{MHz}$ to $7.5 \mathrm{GHz}$, thus UWB system can achieve high-speed transmission, as high as several hundred $\mathrm{Mb} / \mathrm{s}$ to $1 \mathrm{~Gb} / \mathrm{s}$ in the short distance, even with high signal-to-noise ratio (SNR). Fig. 1 shows the comparison between the UWB and narrowband signals.

\footnotetext{
*Yanhan Zeng: yanhanzeng@gzhu.edu.cn
} 


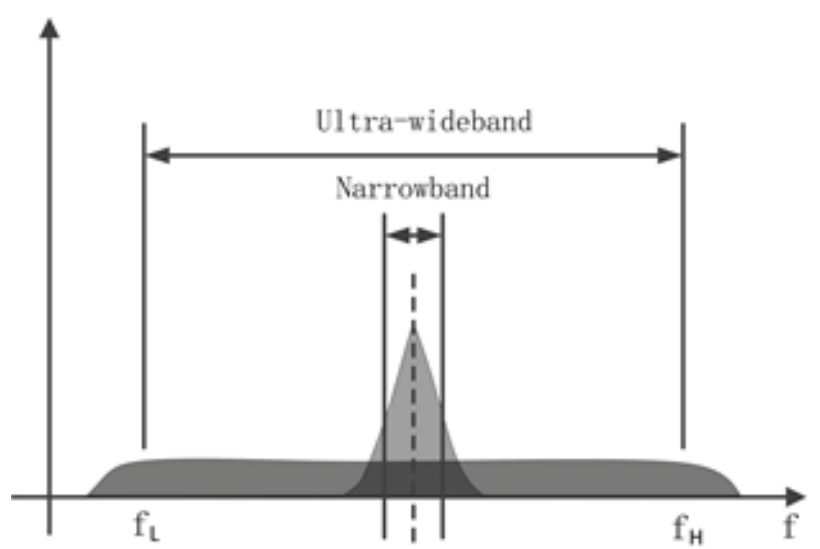

Fig.1. The comparison between the UWB and narrowband signals.

UWB system directly uses ultra-narrow pulse to excitation antenna without carrier. It is easy for the pulse genera- tor to generate the bell-shaped pulse, which is similar to the shape of the Gauss function wave. Thus, the most commonly pulse waveform for the UWB system is the Gauss pulse, and expression of the Gauss pulse in the time domain is:

$$
f(t)= \pm \frac{\sqrt{2}}{a} \exp \left(-\frac{2 \pi t^{2}}{a^{2}}\right)
$$

where $\alpha^{2}=4 \pi \sigma^{2}$ is the pulse factor, and $\sigma 2$ is the variance. The pulse factor determines the width of the pulse. The smaller the pulse factor, the narrower the pulse width will be, and vice versa. The pulse is usually designed to be as a non- current component, which can increase the pulse radiation. The second derivative of Gaussian pulse is mostly used as UWB signal waveform.

The UWB signal transmitting module in this paper is implemented based on the DW1000 chip, which supports the IEEE 802.15.4-2011 UWB standard. The system uses the bi nnary phase modulation (BPSK) and the expression of BPSK is given by:

$$
s(t)=\sum_{k=-\infty}^{\infty} b_{k} p\left(t-k T_{f}\right)
$$

where $b k \in\{-1,1\}$ is the modulation signal, $p(t)$ is the pulse signal and $T f$ is the pulse period.

Table 1. Poll message

\begin{tabular}{|c|c|c|c|}
\hline 21 & 22 & 23 & 24 and up \\
\hline 1 & 1 octet & 1 octet & 0 to 88 octets \\
\hline function & temp & vbat & optional user payload \\
\hline 0x21 & OxTT & 0xVV & - \\
\hline
\end{tabular}

Table 2. Response message

\begin{tabular}{|l|l|l|l|l|}
\hline 21 & 22 & 23 & 24 & 25 and up \\
\hline 1 octet & 1 octet & 1 octet & 1 octet & 0 to 88 octets \\
\hline $\begin{array}{l}\text { functio } \\
\mathrm{n} \\
\text { ande }\end{array}$ & $\begin{array}{l}\text { activity } \\
\text { activity }\end{array}$ & $\begin{array}{l}\text { send ToF } \\
\text { report }\end{array}$ & $\begin{array}{l}\text { activity } \\
\text { parameter }\end{array}$ & $\begin{array}{l}\text { optional user } \\
\text { payload }\end{array}$ \\
\hline 0x10 & $0 \mathrm{x} 02$ & $0 \mathrm{x} 01$ & $0 \mathrm{x} 00$ & - \\
\hline
\end{tabular}

Table 3. Final message

\begin{tabular}{|l|l|l|l|l|}
\hline 21 & 22 to 26 & 27 to 31 & 32 to 36 & 37 and up \\
\hline
\end{tabular}

\begin{tabular}{|l|l|l|l|l|}
\hline $\begin{array}{l}1 \text { octet } \\
1 \text { octet }\end{array}$ & $\begin{array}{l}5 \text { octet } \\
5 \text { octet }\end{array}$ & $\begin{array}{l}5 \text { octet } \\
5 \text { octet }\end{array}$ & $\begin{array}{l}5 \text { octet } \\
5 \text { octet }\end{array}$ & $\begin{array}{l}0 \text { to } 88 \\
\text { octets }\end{array}$ \\
\hline $\begin{array}{l}\text { function } \\
\text { code }\end{array}$ & $\begin{array}{l}\text { poll } \\
\text { message } \\
\text { TX time } \\
\text {-stamp }\end{array}$ & $\begin{array}{l}\text { response } \\
\text { message } \\
\text { RX time } \\
\text {-stamp }\end{array}$ & $\begin{array}{l}\text { embedded fredicted final } \\
\text { TX time } \\
\text {-stamp }\end{array}$ & $\begin{array}{l}\text { optional } \\
\text { user payload }\end{array}$ \\
\hline $0 \times 29$ & - & - & - & - \\
\hline
\end{tabular}

\subsection{Ranging method}

The signal frame is used in this paper for the communication transmission between the mobile node and the fixed base stations. Since the positioning system needs at least three fixed base stations to carry out the distance for the mobile node, four different frame structures, poll message, response message, final message and report message, are used for the handshake communications between base stations and mobile node. The first is the poll message, as shown in the table 1 . Poll message can only be sent by the mobile node for the first communication, which is used to initialize the ranging method. Response message is sent by the fixed base stations. Once receive Poll message, the stations will send Response message, which includes the function code of $0 \times 21$ and a string of 8 bits ToF information, as shown in table 2 . The final message includes three sets of 8-bit timestamps and optional user payload. Report message is similar to the information structure of the Poll message, but it contains four calculated timestamps. The ranging process can be regarded as the three handshakes protocol between the mobile node and the base stations. The well ranging requires precise TX and $\mathrm{RX}$ in- formation. When the base station communicates with the mobile node, it needs to maintain a good synchronization. The ranging process is as shown in the fig. 2 .

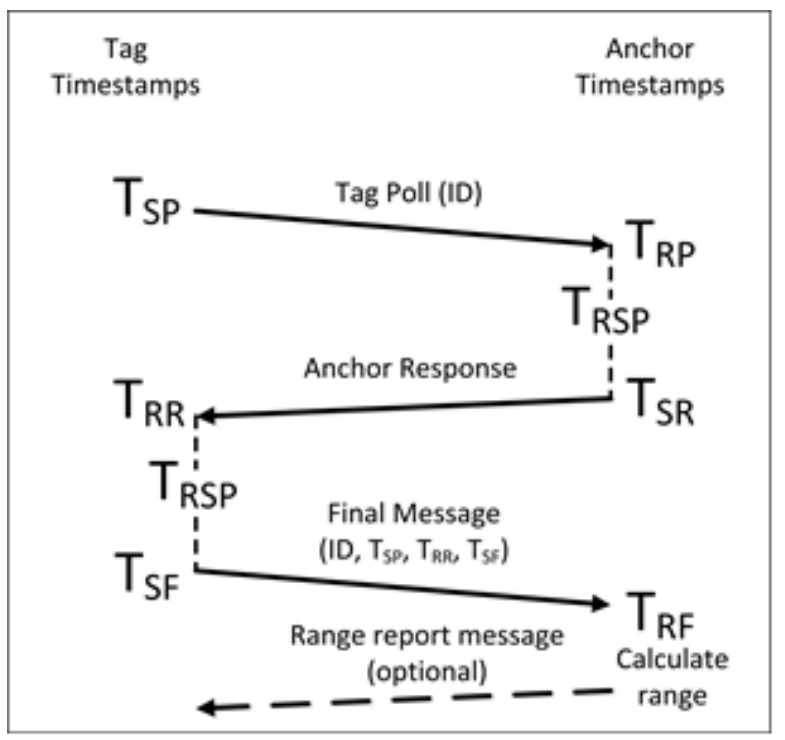

Fig.2. The ranging process.

Each handshake process contains an event stamp. When the handshake process finishes, the time of flight (TOF) is obtained by calculating all the timestamps. 


$$
\mathrm{TOF}=\frac{1}{4}\left(2 \mathrm{~T}_{\mathrm{RR}}-\mathrm{T}_{\mathrm{SP}}-2 \mathrm{~T}_{\mathrm{SR}}+\mathrm{T}_{\mathrm{RP}}+\mathrm{T}_{\mathrm{RF}}-\mathrm{T}_{\mathrm{SF}}\right)
$$

The distance between the mobile node and the fixed base station can be obtained by multiplying TOF with the speed of light.

\section{The improved positioning algorithm}

\subsection{ToA}

The positioning algorithm used in this paper is based on ToA, which is a time delay positioning method. All arrival delay is the propagation time between signals. The positioning can be achieved by calculating the distance between the mobile node and the base station. Making multiple circles by using the fixed base stations as the centers and the measured distances as the radius, the multiple circles has an intersection and the intersection point is the theory position of the mobile node. To achieve this position, at least three rounds are needed, that is why three fixed base stations are needed, as shown in Fig.3. According to Fig.3, the position equations can be obtained as:

$$
d_{i}=\sqrt{\left(x_{i}-x\right)^{2}+\left(y_{i}-y\right)^{2}}
$$

where $(x, y),\left(x_{i}, y_{i}\right)(i=1,2,3)$ and $d i$ are the position of the mobile node and the fixed base stations and the measured distances, respectively. The equation is not a set of nonlinear equations, but they can be reduced to a linear equation group and obtain the solution $(\mathrm{x}, \mathrm{y})$.

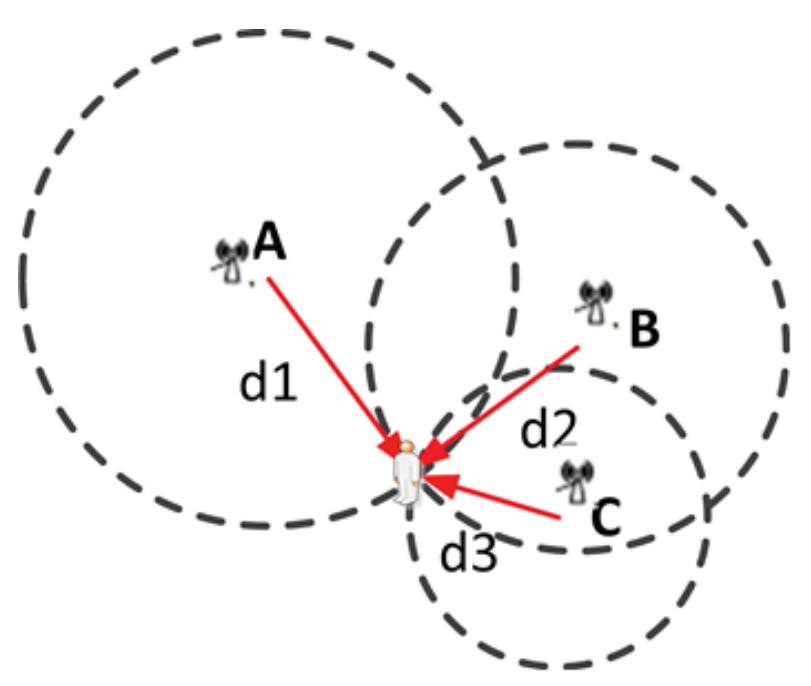

Fig.3. The diagram of ToA

It is necessary to establish an index to correctly evaluate the positioning accuracy. The index used in this paper is the root-mean-square error (RMSE), which is a frequently used measure of the differences between predicted values and the measured values. In the actual measurement, since the number of observations is always limited, the measured value can only be replaced by the most accessible value and the square root error is very sensitive to the large or small error in a set of measurements. because RMSE is the square root of the mean square error, RMSE can well reflect the accuracy of the measurement. RMSE of the positioning accuracy is given by:

$$
\operatorname{RMSE}=\mathrm{E} \sqrt{(x-\hat{x})^{2}+(y-\hat{y})^{2}}
$$

\subsection{Kalman filtering}

As the excellent predictability, Kalman filtering is used to improve the positioning accuracy in this paper. Kalman filtering is an algorithm that optimally estimates the state of the system by using a series of measurements observed based on linear system state equation. Because the measurements observed contain statistical noise and other inaccuracies, the optimal estimation can also be considered as a filtering pro- cess. The state and measurement functions of the Kalman filtering are as follows:

$$
\begin{aligned}
& s(k+1)=A s(k)+\Gamma w(k) \\
& \mathrm{z}(\mathrm{k})=\mathrm{G}(\mathrm{k}) \mathrm{s}(\mathrm{k})+\mathrm{v}(\mathrm{k})
\end{aligned}
$$

From Eq. 7 and Eq. 8, the states at $\mathrm{k}$ time and $\mathrm{k}+1$ time

are linked by the state function. Therefore, with the given

initial values of the state vector and the estimation error, the real-time of state vector can be estimated by the iteration. There are two steps in the iteration of Kalman filter. The first is the prediction of the state as follows:

$$
\begin{aligned}
& \hat{\mathrm{s}}\left(\frac{\mathrm{k}}{\mathrm{k}-1}\right)=\mathrm{As}(\mathrm{k}-1) \\
& \mathrm{P}\left(\frac{\mathrm{k}}{\mathrm{k}-1}\right)=\mathrm{AP}(\mathrm{k}-1) \mathrm{A}^{\mathrm{T}}+\Gamma \mathrm{Q}(\mathrm{k}) \Gamma^{\mathrm{T}} \\
& \mathrm{K}(\mathrm{k})=\mathrm{P}\left(\frac{\mathrm{k}}{\mathrm{k}-1}\right) \mathrm{G}(\mathrm{k})^{\mathrm{T}}\left[\mathrm{G}(\mathrm{k}) \mathrm{P}\left(\frac{\mathrm{k}}{\mathrm{k}-1}\right) \mathrm{G}(\mathrm{k})^{\mathrm{T}}+\mathrm{R}(\mathrm{k})\right]^{-1}
\end{aligned}
$$

The second is the correction of the measured values.

$$
\begin{aligned}
& \hat{\mathrm{s}}(\mathrm{k})=\hat{\mathrm{s}}\left(\frac{\mathrm{k}}{\mathrm{k}-1}\right)+\mathrm{K}(\mathrm{k})\left[\mathrm{z}(\mathrm{k})-\mathrm{G}(\mathrm{k}) \hat{\mathrm{s}}\left(\frac{\mathrm{k}}{\mathrm{k}-1}\right)\right] \\
& \mathrm{P}(\mathrm{k})=[\mathrm{I}-\mathrm{K}(\mathrm{k}) \mathrm{G}(\mathrm{k})] \mathrm{P}\left(\frac{\mathrm{k}}{\mathrm{k}-1}\right)
\end{aligned}
$$

where $\mathrm{s}^{\wedge}(\mathrm{k} / \mathrm{k})$ and $\mathrm{s}^{\wedge}((\mathrm{k}+1) / \mathrm{k})$ are the state estimation and state prediction vectors, respectively, $\mathrm{P}(\mathrm{k} / \mathrm{k})$ and $\mathrm{P}((\mathrm{k}+1) / \mathrm{k})$ are the covariance matrix of estimation error and forecast error, respectively, and $\mathrm{K}(\mathrm{k})$ represents the Kalman gain. As shown in the Fig. 4, compared with the traditional ToA, the simulated RMSE becomes much better for the improved ToA with Kalman filtering. RMSE is close to 0 when the sample increases to 100. The simulation results in Fig. 5 also shows that the improved ToA with Kalman filtering has a better performance in the field of mobile node tracking compared to the traditional ToA, which means that the accuracy is improved. Besides, the measured values tend to be stable when the sample increases. 


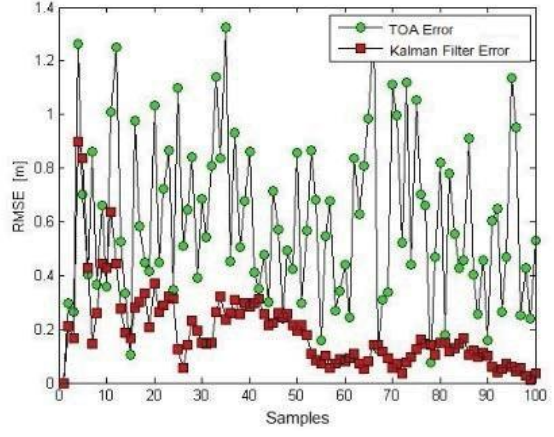

Fig.4. RMSE simulation.

\section{Result and Disscussion}

To verify the performance of proposed indoor positioning system, UWB system contained a mobile node and three base stations is considered. The experiment is done in a $13 \mathrm{~m} * 7.6 \mathrm{~m}$ environment. The mobile node is marked as T0.

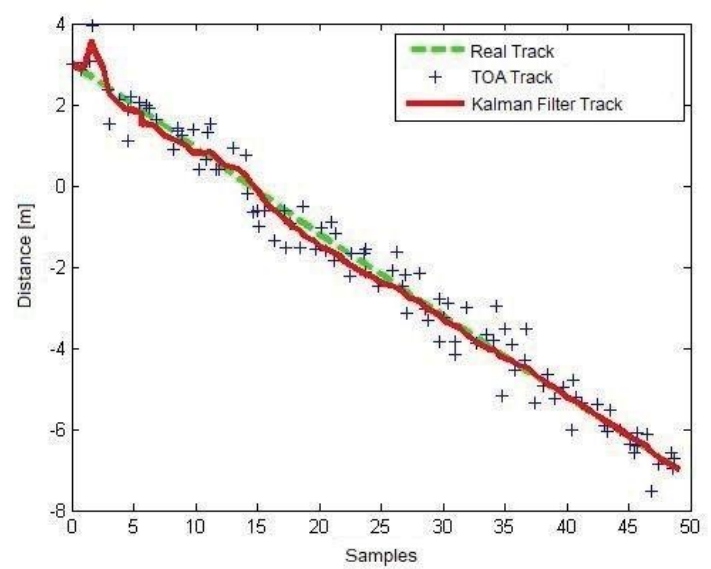

Fig.5. Tracking simulation.

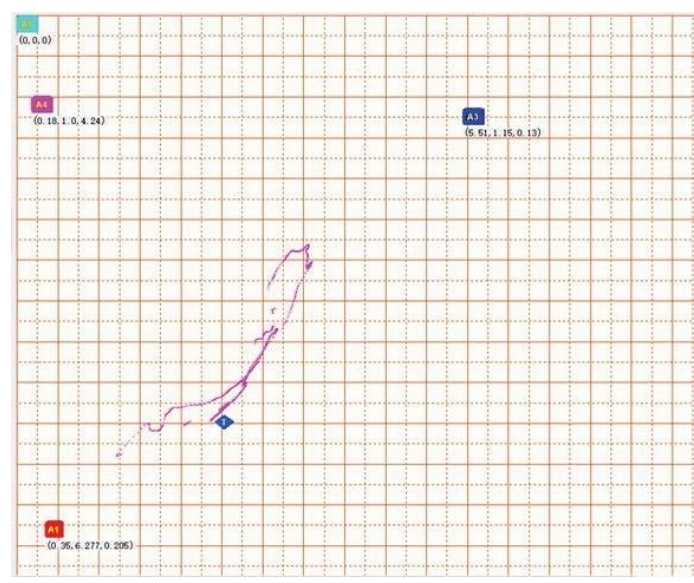

Fig.6. The motion trajectory.

The three fixed base stations are marked as A1, A3 and A4. As shown in the Fig. 6, the purple line is the trajectory of the mobile node and the experiments are conducted in a complex indoor environment with many obstacles. From the measurement results, a small turn of the mobile node can be displayed in the motion trajectory, thus proposed indoor positioning system based on UWB has excellent properties. Besides, when the communication is blocked by the obstacles, the not line of sight (NLOS) error is generated, resulting in the small fluctuations in the motion trajectory.

In the experiment, the true position of the mobile node is measured and compared with the estimated position by the system to calculate the RMSE. As shown in Fig. 7, the positioning error of proposed indoor positioning

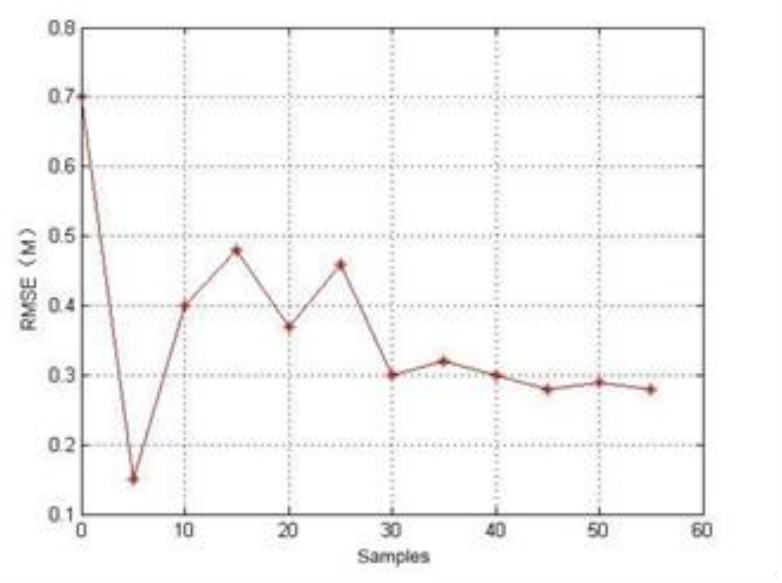

Fig.7. Measured RMSE.

system based on UWB is less than $0.3 \mathrm{~m}$ when the sample is larger than 40 , which can meet the needs of the indoor position very well.

\section{Conclusion}

A high-accuracy indoor positioning system is presented and measured in this paper. UWB technique is used as the ability to penetrate obstacles and fine ranging and the ranging process is simplified to reduce the complexity. Particular attention has been taken to improving the ToA algorithm by utilizing the Kalman filtering, and an outstanding measured RMSE of $0.3 \mathrm{~m}$ is achieved. Owning good performance, proposed system shows its superiority in the indoor location-based service.

\section{ACKNOWLEDGMENT}

This work was supported by the Science and Technology Project of Guangzhou(201804010464), National Natural Science Foundation of China(61704037) and Natural Science Foundation of Guangdong Province, China(2017A030310655).

\section{References}

1. N. A. Alsindi, B. Alavi, and K. Pahlavan. 2009. Mea- surement and Modeling of Ultrawideband TOA-Based Ranging in Indoor Multipath Environments. IEEE Transactions on Vehicular Technology 58, 3 (March 2009), 1046-1058. https://doi.org/10.1109/TVT.2008.926071 
2. P. Bellavista, A. Kpper, and S. Helal. 2008. Location-Based Services: Back to the Future. IEEE Pervasive Computing 7, 2 (April 2008), 85-89. https://doi.org/10.1109/MPRV.2008.34

3. E. DiGiampaolo and F. Martinelli. 2014. Mobile Robot Local- ization Using the Phase of Passive UHF RFID Signals. IEEE Transactions on Industrial Electronics 61, 1 (Jan 2014), 365-376. https://doi.org/10.1109/TIE.2013.2248333

4. Y. Gu, A. Lo, and I. Niemegeers. 2009. A survey of indoor positioning systems for wireless personal networks. IEEE Communications Surveys Tutorials 11, 1 (First 2009), 13-32. https://doi.org/10.1109/SURV.2009.090103

5. S. Hara, D. Anzai, T. Yabu, K. Lee, T. Derham, and R. Zemek.2013. A Perturbation Analysis on the Performance of TOA and TDOA Localization in Mixed LOS/NLOS Environments. IEEE Transactions on Communications 61, 2 (February 2013), 679-689.

https://doi.org/10.1109/TCOMM.2013.012313.1105 09

6. J. He, Y. Geng, F. Liu, and C. Xu. 2014. CC-KF: Enhanced TOA Performance in Multipath and NLOS Indoor Extreme Environment. IEEE Sensors Journal 14, 11 (Nov 2014), 3766-3774. https://doi.org/10.1109/JSEN.2014.2328353

7. Q. Jiang, Y. Ma, K. Liu, and Z. Dou. 2016. A Probabilistic Radio Map Construction Scheme for Crowdsourcing-Based Fingerprinting Localization. IEEE Sensors Journal 16, 10 (May 2016), 37643774. https://doi.org/10.1109/JSEN.2016.2535250

8. R. Kumar, J. C. Cousin, B. Huyart, and K. Mabrouk. 2013. 2D measurement using interferometry and FMCW multistatic radar system for indoor localization. In 2013 European Radar Conference. 17-20.

9. R. H. Lee, J. C. Wu, S. H. Chang, S. F. Chang, C. C. Chang, and Y. M. Chen. 2013. Radar design for wireless indoor positioning applications. In 2013 European Microwave Conference. 846-849. https://doi.org/10.23919/EuMC.2013.6686789

10. R. K. Mahapatra and N. S. V. Shet. 2016. Experimental analysis of RSSI-based distance estimation for wireless sen- sor networks. In 2016 IEEE Distributed Computing, VL-SI, Electrical Circuits and Robotics (DISCOVER). 211-215. https://doi.org/10.1109/DISCOVER.2016.7806221

11. Achraf Mallat, J'er^ome Louveaux, Luc Vandendorpe, Mario Di Dio, and Marco Luise. 2012. Discrete fourier transform-based TOA estimation in UWB systems. EURASIP Journal on Wireless Communications and Networking 2012, 1 (2012), 3.

12. K. Pahlavan, Xinrong Li, and J. P. Makela. 2002. Indoor geolocation science and technology. IEEE Communications Magazine 40, 2 (Feb 2002), 112118. https://doi.org/10.1109/35.983917

13. A. Wille, M. Broll, and S. Winter. 2011. Phase difference-based RFID navigation for medical applications. In 2011 IEEE International Conference on RFID. 98-105. https://doi.org/10.1109/RFID.2011.5764608 